doi:10.1017/\$1041610218001862

\section{Patient with Parkinson's disease presenting with impulse control disorders following treatment with selegiline}

Impulse control disorders (ICDs) in patients with Parkinson's disease (PD) often affect quality of life and pose a serious burden for relatives. Such behavioral disorders typically manifest as pathological gambling, hypersexuality, compulsive spending, shopping, or eating (American Psychiatric Association, 1994). These behaviors were identified in $17.1 \%$ of patients treated with dopamine agonists, compared with only $6.9 \%$ of those not treated with these drugs in a study of over 3000 patients with PD (Weintraub et al., 2010). However, only a few preliminary studies appear to have investigated the relationship between ICDs and monoaminoxidase-B (MAO-B) inhibitors (Perez-Lloret et al., 2012).

We report a case of a 71-year-old man who showed compulsive sexual behavior and punding for seven months after commencing treatment with selegiline. He had first presented three years earlier with bradykinesia, gait disorder, and bilateral muscular rigidity of the upper and lower limbs, which resulted in a diagnosis of PD. At that time, he showed no cognitive dysfunction or comorbidities, including psychiatric disease, and met the UK Parkinson's Disease Society Brain Bank Clinical Diagnostic Criteria. In brain imaging, 123I-2ß-Carbomethoxy-3ß-(4-iosophwnyl)$\mathrm{N}$-(3-fluoropropyl)-N-(3-fluoropropyl) nortropane (123I-FP-CIT) dopamine transporter single photon emission computed tomography (DAT SPECT) revealed a significant reduction in DAT uptake in the bilateral basal ganglia, but CT showed no infarctions. Later, anti-Parkinson's drugs, such as levodopa (36 months previously), ropinirole (35 months previously), and selegiline (29 months previously) were prescribed. His initial symptom rating had been a grade 3 based on the Hoehn and Yahr scale. The first compulsive sexual behavior that his wife noticed was his entering the bathroom without permission while she was taking a bath. His symptoms subsequently gradually worsened and came to include watching porn videos and punding (getting absorbed in puzzles). On visiting our department again seven months after these symptoms first appeared, the behavioral side effects of selegiline were suspected, as this was the drug most recently prescribed among the list given above. It was decided at this point to discontinue selegiline. His compulsive sexual behavior and punding disappeared immediately after this and were not observed for eight months thereafter. Therefore, the final diagnosis based on the Diagnostic and Statistical Manual of Mental Disorders (Fourth Edition) (DSM-4) was iatrogenic ICDs (American Psychiatric Association, 1994).

Although the underlying mechanisms of ICDs in PD remain to be fully clarified, dysregulation of the mesocorticolimbic dopamine system is believed to be a major neurobiological substrate for these behaviors (Weintraub and Claassen, 2017). Although there are few case reports on selegiline-induced ICDs in PD, MAO-B inhibitors have been known to increase central dopaminergic tone, which could possibly affect such mesocorticolimbic dopamine systems (Rascol, 2005). Furthermore, selegiline has been reported to include a methamphetamine metabolite, which might also contribute to the development of addictive behaviors (Weintraub et al., 2010; Rascol, 2005). This suggests that selegiline may have been the prime inducer of compulsive sexual behavior and punding in the present patient, as his symptoms showed a marked improvement upon its withdrawal. It is possible, however, that its combination with other drugs, such as levodopa and dopamine agonists, underlies the apparent increase in risk of ICDs with this drug.

Impulse control disorders should always be considered as a possibility in PD patients treated with selegiline, as well as with dopamine agonists, and should not be overlooked. They could be treatable under these conditions simply by withdrawal or a reduction in other anti-Parkinson's drugs.

\section{Conflict of interest declaration}

The authors have no financial disclosures to declare. There are no conflicts of interest regarding this letter.

\section{Description of authors' roles}

Study concept and design: K. Hirao, H. Hanyu. Acquisition of subjects and/or data: K. Hirao, Y. Kaneko, D. Hirose, R. Fukasawa, S. Soichiro, H. Kanetaka, T. Umahara, H. Sakurai, H. Hanyu. Analysis and interpretation of data: K. Hirao, H. Hanyu.

Preparation of manuscript: Hirao, H. Hanyu. 


\section{Acknowledgments}

We would like to thank Professor Jeremy Williams at the Department of International Medical Communications at Tokyo Medical University for reviewing the manuscript.

\section{References}

American Psychiatric Association (1994). Diagnostic and Statistical Manual of Mental Disorders, 4th ed. Washington, DC: American Psychiatric Association.

Perez-Lloret, S., et al. (2012). Prevalence and pharmacological factors associated with impulse-control disorder symptoms in patients with Parkinson disease. Clinical Neuropharmacology, 35, 261-265. doi: 10.1097/ WNF.0b013e31826e6e6d.

Rascol, O. (2005). Rasagiline in the pharmacotherapy of Parkinson's disease- a review. Expert Opinion on Pharmacotherapy, 6, 2061-2075. doi: 10.1517/14656566.6.12.2061.

Weintraub, D., et al. (2010). Impulse control disorders in Parkinson disease: a cross-sectional study of 3090 patients. Archives of Neurology, 67, 589-595. doi: 10.1001/ archneurol.2010.65.

Weintraub, D. and Claassen, D. O. (2017). Impulse control and related disorders in Parkinson's disease. International Review of Neurobiology, 133, 679-717. doi: 10.1016/bs.irn.2017.04.006.

Kentaro Hirao, Yoshitsugu Kaneko, Daisuke Hirose, Raita Fukasawa, Soichiro Shimizu, Hidekazu Kanetaka, Takahiko Umahara, Hirofumi Sakurai and Haruo Hanyu

Department of Geriatric Medicine, Tokyo Medical University, Tokyo, Japan

Correspondence should be addressed to: Kentaro Hirao, Department of Geriatric Medicine, Tokyo Medical University, 6-7-1 Nishi-Shinjuku, Shinjukuku, Tokyo 160-0023, Japan. Email: kentaroh@, tokyo-med.ac.jp. Phone: +81-3-3342-6111; Fax: +81-3-3342-2305. 